\title{
AN IDENTITY IN JORDAN RINGS
}

\author{
MARSHALL HALL, JR.
}

1. Introduction. An abstract Jordan ring $J$ is a distributive ring in which multiplication satisfies the two laws

$$
\begin{aligned}
b a & =a b, \\
\left(a^{2} b\right) a & =a^{2}(b a) .
\end{aligned}
$$

We shall assume here that division by two is possible, or more precisely that $x+x=b$ has a unique solution $x$ for any given $b$. This will of course be true if $J$ is an algebra over a field of characteristic different from 2. Jacobson [5] has defined the Jordan triple product $\{a b c\}$ by the rule

$$
\{a b c\}=\frac{1}{2}(a b) c+\frac{1}{2}(b c) a-\frac{1}{2}(a c) b .
$$

A special Jordan ring $S$ may be formed from an associative ring $R$, by taking the elements of $R$ with the same addition but with a Jordan product $a b$ given in terms of the associative product $a \cdot b$ of $R$ by the rule

$$
a b=a \cdot b+b \cdot a .
$$

From (1.4) the laws (1.1) and (1.2) follow and the triple product simplifies to the form

$$
\{a b c\}=a \cdot b \cdot c+c \cdot b \cdot a .
$$

In this paper the identity

$$
\{a b a\}^{2}=\left\{a\left\{b a^{2} b\right\} a\right\}
$$

is proved to hold in abstract Jordan rings. This is immediate for special Jordan rings, but was posed as an unsolved problem by Jacobson in his Colloquium lectures at Ann Arbor in September, 1955. In this paper the identity is proved by finding a partial basis for the free Jordan ring with two generators, the basis being found for all elements of degree at most 5 and for elements of degree 4 in $a$ and degree 2 in $b$. Expressing both sides of (1.6) in terms of the basis, the two sides are found to be identical.

Using this identity, Jacobson [5] has been able to prove certain structure and representation theorems for abstract Jordan algebras which had been proved previously by Albert [1] for special algebras.

Received by the editors January 24, 1956. 
A further interesting by-product of this paper is that, so far as it goes, the basis indicates that the free Jordan ring is special. This comes from a comparison with results established by P. M. Cohn [4].

Another proof of the identity has been found independently by Mr. Lawrence Harper of the University of Chicago. He does not find a basis, but uses a sequence of relations which are consequences of (1.1) and (1.2).

2. The monomial relations of a Jordan ring. In a Jordan ring addition is an abelian group, both distributive laws hold and the multiplication must satisfy the two laws

$$
\begin{aligned}
x y & =y x, \\
\left(y^{2} x\right) y & =y^{2}(x y) .
\end{aligned}
$$

We assume also that division by 2 is possible, or more precisely that the additive group contains no element of order 2. This will of course be true if the ring admits as operators a field of characteristic different from 2 .

In a ring generated by elements $a_{1}, \cdots, a_{n}$ every element can be expressed as a linear combination of monomials, and to find all relations, it is sufficient to find the relations on the monomials. It is trivial to verify that the commutative law (2.1) will hold for all elements if it holds for all monomials.

In (2.2) replace $y$ by $y+z$ and from the result subtract both (2.2) and (2.2) with $y$ replaced by $z$. This gives

$$
\begin{aligned}
& \left(y^{2} x\right) z+2((y z) x) y+2((y z) x) z+\left(z^{2} x\right) y \\
& \quad=y^{2}(x z)+2(y z)(x y)+2(y z)(x z)+z^{2}(x y) .
\end{aligned}
$$

In (2.3) replace $z$ by $z+w$ and from this subtract both (2.3) and (2.3) with $z$ replaced by $w$. Dividing by 2 we get

$$
\begin{aligned}
(x(y z)) w+ & (x(y w)) z+(x(z w)) y \\
& =(x w)(y z)+(x z)(y w)+(x y)(z w) .
\end{aligned}
$$

With $w=y$ and $w=z$ we may derive (2.3) from (2.4), while if $w=z=y$ (2.4) becomes three times (2.2). Using induction on the number of monomials involved, it is easy to show that (2.1) and (2.2) hold for all elements if and only if (2.1), (2.2) and (2.4) hold for all monomials. We note that (2.4) is symmetric in $y, z, w$.

3. Basis elements in the free Jordan ring with two generators. We shall find a basis for elements of degrees one through five in the free Jordan ring $J$ generated by two elements $a$ and $b$. Since (2.2) and (2.4) do not apply below the fourth degree, we can write down a basis for the first three degrees immediately. 


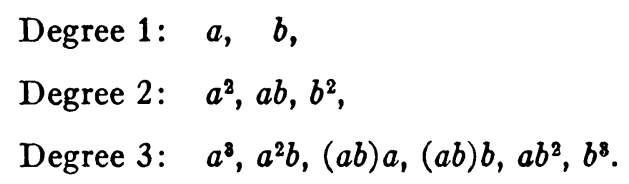

Here we have written $a^{2} a=a^{3}$ and $b^{2} b=b^{8}$. It is known that a Jordan ring is power associative. If we write $a^{1}=a$, and recursively $a^{n}=a^{n-1} a$, then it will be true that if $t+u=n$ then $a^{z} a^{u}=a^{n}$, and the ring generated by $a$ is associative as well as commutative. From (2.2) with $x=a^{n-3}, y=a$ we get $\left(a^{n-3} a^{2}\right) a=\left(a^{n-2}\right) a^{2}$ and so by induction $\left(a^{n-2}\right) a^{2}$ $=a^{n}$. If in (2.4) we put $x=a^{s}, y=a^{r}, z=w=a$, we obtain $2\left(a^{r+s+1}\right) a$ $+\left(a^{s+2}\right) a^{r}=2\left(a^{s+1}\right) a^{r+1}+\left(a^{s+r}\right) a^{2}$. By taking appropriate values for $s$ and $r$ we may complete the relations $a^{n}=a^{n-1} a=a^{n-2} a^{2}=a^{n-3} a^{3}$ $=\cdots=a^{t} a^{u}$, naturally using induction on $n$, and one of $s$ or $r$.

For degree 4 with each of $x$ and $y$ taking the values $a$ and $b$ in (2.2) we get the four relations

$$
\begin{array}{ll}
\left(a^{2} a\right) a=a^{2} a^{2}=a^{4}, & \left(b^{2} b\right) b=b^{2} b^{2}=b^{4}, \\
\left(b^{2} a\right) b=b^{2}(a b), & \left(a^{2} b\right) a=a^{2}(b a) .
\end{array}
$$

From (2.4) we obtain further relations of degree four.

$$
\begin{array}{llllll}
x & y & z & w & \\
a & a & b & b & 2(a(a b)) b+\left(a b^{2}\right) a=2(a b)^{2}+a^{2} b^{2}, \\
a & b & a & a & 2(a(a b)) a+a^{3} b & =3 a^{2}(a b), \\
b & a & b & b & 2(b(a b)) b+b^{3} a & =3 b^{2}(a b), \\
b & b & a & a & 2(b(a b)) a+\left(a^{2} b\right) b & =2(a b)^{2}+a^{2} b^{2} .
\end{array}
$$

From (3.2) and (3.3) we can now give a basis for degree 4 and express the remaining monomials in terms of this basis.

Degree 4 basis and relations.

$$
\begin{aligned}
& a^{4} ;\left(a^{2} b\right) a,((a b) a) a ;((a b) b) a,((a b) a) b,(a b)^{2}, a^{2} b^{2} ; \\
& \left(a b^{2}\right) b,((a b) b) b ; b^{4} . \\
& a^{3}(a b)=\left(a^{2} b\right) a, b^{2}(a b)=\left(a b^{2}\right) b, \\
& a^{3} b=3\left(a^{2} b\right) a-2((a b) a) a, a b^{3}=3\left(a b^{2}\right) b-2((a b) b) b \\
& \left(a b^{2}\right) a=a^{2} b^{2}+2(a b)^{2}-2((a b) a) b \\
& \left(a^{2} b\right) b=a^{2} b^{2}+2(a b)^{2}-2((a b) b) a .
\end{aligned}
$$


In finding a basis for degree 5 , it is convenient to label the monomials:

$$
\begin{array}{ll}
u_{1}=(((a b) a) a) a, & v_{1}=(((a b) b) a) a, \\
u_{2}=\left(\left(a^{2} b\right) a\right) a, & v_{2}=(((a b) a) b) a, \\
u_{3}=a^{4} b, & v_{3}=\left(a^{2} b^{2}\right) a, \\
u_{4}=((a b) a) a^{2}, & v_{4}=(a b)^{2} a, \\
u_{5}=\left(a^{2} b\right) a^{2}, & v_{5}=(((a b) a) a) b, \\
u_{6}=a^{3}(a b), & v_{6}=\left(\left(a^{2} b\right) a\right) b, \\
u_{7}=\left(a^{2}(a b)\right) a=u_{2}, & v_{7}=\left(a b^{2}\right) a^{2}, \\
u_{8}=\left(a^{8} b\right) a=-2 u_{1}+3 u_{2}, & v_{8}=((a b) b) a^{2}, \\
& v_{10}=((a b) a)(a b), \\
& v_{12}=\left(\left(a b^{2}\right) a\right) a=-2 v_{2}+v_{3}+2 v_{4}, \\
& v_{13}=\left(\left(a^{2} b\right) b\right) a=-2 v_{1}+v_{3}+2 v_{4}, \\
& v_{14}=\left(a^{2}(a b)\right) b=v_{6},
\end{array}
$$

The relations here are derived from (3.5) as may be easily verified. There will also be the monomials obtained by interchanging $a$ and $b$ in the above list, as well as the monomials $a^{5}$ and $b^{5}$. Substituting in (2.2) we find

$$
\begin{aligned}
& x=a b, y=a, \quad u_{4}=u_{7}, \\
& x=b^{2}, \quad y=a, \quad v_{7}=v_{3} \text {. }
\end{aligned}
$$

The law (2.4) gives the following relations on the $u$ 's:

$$
\begin{array}{lllll}
x & y & z & w & \\
a^{2} & a & a & b & u_{3}+2 u_{7}=u_{5}+2 u_{6}, \\
a & a^{2} & a & b & u_{2}+u_{3}+u_{4}=u_{5}+2 u_{6}, \\
a & a b & a & a & u_{6}+2 u_{1}=3 u_{4}, \\
b & a^{2} & a & a & u_{5}+2 u_{8}=u_{5}+2 u_{6} .
\end{array}
$$

We also find from (2.4) the following relations on the $v$ 's. 


$$
\begin{array}{lllll}
x & y & z & w & \\
a^{2} & b & b & a & v_{3}+2 v_{14}=v_{11}+2 v_{10}, \\
a b & a & a & b & v_{14}+2 v_{4}=v_{8}+2 v_{9}, \\
a & a^{2} & b & b & 2 v_{6}+v_{7}=2 v_{10}+v_{11}, \\
a & a b & a & b & v_{5}+v_{9}+v_{1}=v_{8}+2 v_{9}, \\
a & b^{2} & a & a & v_{11}+2 v_{12}=3 v_{7}, \\
b & a^{2} & a & b & v_{15}+v_{13}+v_{8}=2 v_{10}+v_{11}, \\
b & a b & a & a & 2 v_{2}+v_{10}=v_{8}+2 v_{9} .
\end{array}
$$

From these we may find a basis and relations for degree 5 .

Type $a^{5}: \quad a^{5}=a^{4} a=a^{3} a^{2}$.

Type $a^{4} b$ : Basis $u_{1}, u_{2}, u_{3}$.

$$
\begin{aligned}
& u_{4}=u_{2}, \\
& u_{5}=4 u_{1}-4 u_{2}+u_{3}, \\
& u_{6}=-2 u_{1}+3 u_{2}, \\
& u_{7}=u_{2}, \\
& u_{8}=-2 u_{1}+3 u_{2} .
\end{aligned}
$$

Type $a^{3} b^{2}$ : Basis $v_{1}, v_{2}, v_{3}, v_{4}, v_{5}, v_{6}$.

$$
\begin{aligned}
& v_{7}=v_{3}, \\
& v_{8}=2 v_{1}-2 v_{4}+2 v_{5}-v_{6}, \\
& v_{9}=-v_{1}+2 v_{4}-v_{5}+v_{6}, \\
& v_{10}=-2 v_{2}+2 v_{4}+v_{6}, \\
& v_{11}=4 v_{2}+v_{3}-4 v_{4}, \\
& v_{12}=-2 v_{2}+v_{3}+2 v_{4}, \\
& v_{13}=-2 v_{1}+v_{3}+2 v_{4}, \\
& v_{14}=v_{6}, \\
& v_{15}=-2 v_{5}+3 v_{6} .
\end{aligned}
$$

By interchanging $a$ and $b$ throughout, we will also find a basis of 6 elements of type $a^{2} b^{3}, 3$ elements of type $a b^{4}$ and the single element $b^{5}$. Thus there are a total of 20 basis elements of degree five.

4. Basis and relations for terms of type $a^{4} b^{2}$. Since the identity depends on terms which are all of type $a^{4} b^{2}$, it will be sufficient to find a basis and relations for the monomials of this type. Using the relations of (3.4) and (3.9) any element of type $a^{4} b^{2}$ can be expressed in terms 
of the following twenty one monomials:

$$
\begin{aligned}
& x_{1}=((((a b) b) a) a) a=v_{1} a, \quad x_{11}=(((a b) a) b) a^{2}, \\
& x_{2}=((((a b) a) b) a) a=v_{2} a, \quad x_{12}=(a b)^{2} a^{2}, \\
& x_{3}=\left(\left(a^{2} b^{2}\right) a\right) a \quad=v_{3} a, \quad x_{13}=\left(a^{2} b^{2}\right) a^{2}, \\
& x_{4}=\left((a b)^{2} a\right) a \quad=v_{4} a, \quad x_{14}=(((a b) a) a)(a b), \\
& x_{5}=((((a b) a) a) b) a=v_{5} a, \quad x_{15}=\left(\left(a^{2} b\right) a\right)(a b) \text {, } \\
& x_{6}=\left(\left(\left(a^{2} b\right) a\right) b\right) a=v_{6} a, \quad x_{16}=a^{4} b^{2} \text {, } \\
& x_{7}=((((a b) a) a) a) b=u_{1} b, \quad x_{17}=a^{3}\left(a b^{2}\right), \\
& x_{8}=\left(\left(\left(a^{2} b\right) a\right) a\right) b=u_{2} b, \quad x_{18}=a^{8}((a b) b), \\
& x_{9}=\left(a^{4} b\right) b \quad=u_{3} b, \quad x_{19}=((a b) a)^{2}, \\
& x_{10}=(((a b) b) a) a^{2}, \quad x_{20}=\left(a^{2} b\right)((a b) a) \text {, } \\
& x_{21}=\left(a^{2} b\right)^{2} \text {. }
\end{aligned}
$$

We will also label the remaining monomials and express them in terms of the above list.

$$
\begin{aligned}
& x_{22}=\left(\left(a b^{2}\right) a^{2}\right) a \quad=v_{7} a=x_{3}, \\
& x_{23}=\left(((a b) b) a^{2}\right) a=v_{8} a=2 x_{1}-2 x_{4}+2 x_{5}-x_{6} \text {, } \\
& x_{24}=(((a b) a)(a b)) a=v_{9} a=-x_{1}+2 x_{4}-x_{6}+x_{6} \text {, } \\
& x_{25}=\left(\left(a^{2} b\right)(a b)\right) a=v_{10} a=-2 x_{2}+2 x_{4}+x_{6} \text {, } \\
& x_{26}=\left(a^{3} b^{2}\right) a \quad=v_{11} a=4 x_{2}+x_{3}+-4 x_{4} \text {, } \\
& x_{27}=\left(\left(\left(a b^{2}\right) a\right) a\right) a=v_{12} a=-2 x_{2}+x_{3}+2 x_{4} \text {, } \\
& x_{28}=\left(\left(\left(a^{2} b\right) b\right) a\right) a=v_{13} a=-2 x_{1}+x_{3}+2 x_{4} \text {, } \\
& x_{29}=\left(\left((a b) a^{2}\right) b\right) a=v_{14} a=x_{6} \text {, } \\
& x_{30}=\left(\left(a^{8} b\right) b\right) a \quad=v_{16} a=-2 x_{5}+3 x_{6} \text {, } \\
& x_{31}=\left(((a b) a) a^{2}\right) b=u_{4} b=x_{8} \text {, } \\
& x_{32}=\left(\left(a^{2} b\right) a^{2}\right) b \quad=u_{5} b=4 x_{7}-4 x_{8}+x_{9}, \\
& x_{33}=\left(a^{3}(a b)\right) b \quad=u_{6} b=-2 x_{7}+3 x_{8} \text {, } \\
& x_{34}=\left(\left(a^{2}(a b)\right) a\right) b=u_{7} b=x_{8} \text {, } \\
& x_{35}=\left(\left(a^{8} b\right) a\right) b \quad=u_{8} b=-2 x_{7}+3 x_{8} \text {, } \\
& x_{36}=\left(\left(a b^{2}\right) a\right) a^{2}=x_{18}+2 x_{12}-2 x_{11} \text {, } \\
& x_{37}=\left(\left(a^{2} b\right) b\right) a^{2}=x_{13}+2 x_{12}-2 x_{10} \text {, } \\
& x_{38}=\left(a^{2}(a b)\right)(a b)=x_{15} \text {, } \\
& x_{39}=\left(a^{8} b\right)(a b)=3 x_{15}-2 x_{14} \text {. }
\end{aligned}
$$


The law (2.2) has only two applications to elements of type $a^{4} b^{2}$

$$
\begin{array}{lll}
x=a b^{2}, & y=a, & x_{22}=x_{36}, \\
x=(a b) b, & y=a, & x_{23}=x_{10} .
\end{array}
$$

There are, however, twenty-two applicable cases of (2.4): $\begin{array}{llll}x & y & z & w\end{array}$
1. $a^{3}, \quad a, \quad b, \quad b, \quad 2 x_{33}+x_{26}=x_{16}+2 x_{39}$,
2. $a^{2} b, b, \quad a, \quad a, \quad 2 x_{25}+x_{32}=2 x_{15}+x_{37}$,
3. $(a b) a, b, \quad a, \quad a, \quad 2 x_{24}+x_{31}=2 x_{14}+x_{11}$,
4. $a^{2}, \quad a^{2}, \quad b, \quad b, \quad 2 x_{32}+x_{13}=2 x_{21}+x_{16}$,
5. $a^{2}, \quad a b, \quad a, \quad b, \quad x_{31}+x_{23}+x_{38}=x_{20}+x_{18}+x_{38}$,
6. $a^{2}, b^{2}, a, \quad a, \quad 2 x_{22}+x_{16}=x_{13}+2 x_{17}$,
7. $a b, \quad a^{2}, a, \quad b, \quad x_{33}+x_{25}+x_{12}=x_{38}+x_{20}+x_{18}$
8. $a b, a b, \quad a, \quad a, \quad 2 x_{24}+x_{38}=x_{12}+2 x_{19}$,
9. $b^{2}, \quad a^{2}, \quad a, \quad a, \quad 2 x_{26}+x_{13}=2 x_{17}+x_{13}$,
10. $a, \quad a^{3}, \quad b, \quad b, \quad 2 x_{36}+x_{17}=x_{16}+2 x_{39}$,
(4.4) 11. $a, \quad a^{2} b, a, \quad b, \quad x_{8}+x_{28}+x_{20}=2 x_{15}+x_{37}$,
12. $a, \quad(a b) a, a, \quad b, \quad x_{7}+x_{2}+x_{19}=2 x_{14}+x_{11}$,
13. $a, a b^{2}, a, a, \quad 2 x_{27}+x_{17}=3 x_{36}$,
14. $a,(a b) b, a, a, \quad 2 x_{1}+x_{18}=3 x_{10}$,
15. $b, \quad a^{3}, \quad a, \quad b, \quad x_{9}+x_{30}+x_{18}=2 x_{39}+x_{16}$,
16. $b, \quad a^{2} b, a, \quad a, \quad 2 x_{6}+x_{21}=2 x_{15}+x_{37}$,
17. $b, \quad(a b) a, a, \quad a, \quad 2 x_{5}+x_{20}=2 x_{14}+x_{11}$,
18. $a, \quad a^{2}, \quad a b, \quad b, \quad x_{34}+x_{16}+x_{10}=x_{18}+x_{20}+x_{38}$,
19. $a, a^{2}, \quad b^{2}, \quad a, \quad x_{3}+x_{16}+x_{36}=2 x_{17}+x_{13}$,
20. $a, a b, a b, a, \quad 2 x_{14}+x_{4}=2 x_{19}+x_{12}$,
21. $b, \quad a^{2}, \quad a^{2}, \quad b, \quad 2 x_{37}+x_{9}=x_{16}+2 x_{21}$,
22. $b, \quad a^{2}, a b, a, \quad x_{29}+x_{39}+x_{11}=x_{20}+x_{18}+x_{38}$.

From these 24 relations we find a basis and express the remaining monomials in terms of this basis. 
Basis: $x_{1}, x_{2}, x_{3}, x_{4}, x_{8}, x_{8}, x_{7}, x_{8}, x_{14}$

$$
\begin{aligned}
& x_{9}=-4 x_{1}+4 x_{2}+x_{3}+2 x_{4}-x_{8}-4 x_{7}+6 x_{8} \\
& x_{10}=2 x_{1} \quad-2 x_{4}+2 x_{3}-x_{8} \\
& x_{11}=-2 x_{1} \quad+4 x_{4}-2 x_{8}+2 x_{0}+x_{8}-2 x_{14} \\
& x_{12}=4 x_{1}+2 x_{2} \quad-7 x_{4}+4 x_{8}-4 x_{6}+2 x_{7}-2 x_{8}+2 x_{14} \text {, } \\
& x_{13}=-12 x_{1}-4 x_{2}+x_{8}+22 x_{4}-12 x_{6}+12 x_{6}-4 x_{7}+6 x_{8}-8 x_{14} \text {, } \\
& \text { (4.5) } \quad x_{16}=2 x_{1} \quad-3 x_{4}+2 x_{6}-2 x_{6} \quad+2 x_{14} \text {, } \\
& x_{16}=-12 x_{1}+4 x_{2}+x_{3}+14 x_{4}-12 x_{6}+12 x_{6}-4 x_{7}+6 x_{8}-8 x_{14} \text {, } \\
& x_{17}=\quad+4 x_{2}+x_{3}-4 x_{4} \text {, } \\
& x_{18}=4 x_{1} \quad-6 x_{4}+6 x_{6}-3 x_{6}, \\
& x_{19}=-2 x_{1}-x_{2}+4 x_{4}-2 x_{6}+2 x_{6}-x_{7}+x_{8} \text {, } \\
& x_{20}=-2 x_{1}+4 x_{4}-4 x_{6}+2 x_{6}+x_{8} \text {, } \\
& x_{21}=-4 x_{1}+x_{8}+6 x_{4}-4 x_{5}+2 x_{8} \text {. }
\end{aligned}
$$

The remaining monomials may of course be expressed in terms of these by using (4.2).

5. Proof of the identity. In a Jordan ring, Jacobson defines a ternary operation $a b c$ by the rule

$$
\{a b c\}=\frac{1}{2}(a b) c+\frac{1}{2}(b c) a-\frac{1}{2}(a c) b .
$$

The identity which we wish to prove is the following:

$$
\{a b a\}^{2}=\left\{a\left\{b a^{2} b\right\} a\right\} .
$$

This is known to hold in special Jordan rings, but here we show that it is true in every Jordan ring. Using (5.1) we express both sides of (5.2) in terms of monomials.

$$
\begin{aligned}
\{a b a\}^{2}= & ((a b) a)^{2}-\left(a^{2} b\right)((a b) a)+\frac{1}{4}\left(a^{2} b\right)^{2} \\
= & x_{19}-x_{20}+\frac{1}{4} x_{21} \\
\left\{a\left\{b a^{2} b\right\} a\right\}= & \left(\left(\left(a^{2} b\right) b\right) a\right) a-\frac{1}{2}\left(\left(a^{2} b^{2}\right) a\right) a-\frac{1}{2}\left(\left(a^{2} b\right) b\right) a^{2} \\
& +\frac{1}{4}\left(a^{2} b^{2}\right) a^{2}=x_{28}-\frac{1}{2} x_{3}-\frac{1}{2} x_{37}+\frac{1}{4} x_{13} .
\end{aligned}
$$

This identity is indeed true since using the relations of (4.2) and (4.4) both expressions reduce to 


$$
-x_{1}-x_{2}+\frac{1}{4} x_{3}+\frac{3}{2} x_{4}+x_{6}-x_{7}+\frac{1}{2} x_{8} .
$$

6. Concluding remarks. ${ }^{1}$ Comparison of the results found here with those of P. M. Cohn [2] is of some interest. In $\$ 3$ we found all relations on the free ring $J$ with two generators for elements of degrees not exceeding five, and from these found the number of basis elements for degrees one through five to be respectively $2,3,6,10,20$. Cohn has shown that the special free ring with two generators has a basis of the reversible elements. This basis will consist of monomials which are their own reverses together with elements which are the sum of a monomial and its reverse, the reverse being distinct. This leads to a basis with $2^{n-1}+2^{r}$ elements of degree $n$ when $n=2 r+1$ or $2 r+2$. Thus from a consideration of the number of basis elements alone, it follows that $J$ modulo the ideal $K_{6}$ of elements of degree six or higher is a special ring, agreeing with the free special ring modulo elements of degree six or higher. In particular, every identity on two generators of degree not exceeding five which holds in a special Jordan ring also holds in any Jordan ring with two generators. Similar considerations apply if we include also the elements of degree four in one variable and two in the other since there will be nine reversible basis elements of this type in the free special Jordan ring. To prove that the free Jordan ring with two generators is special it would be sufficient to show that for $n=2 r+1$ or $2 r+2$ there are at most $2^{n-1}+2^{r}$ basis elements of degree $n$.

\section{BIBLIOGRAPHY}

1. A. A. Albert, On Jordan algebras of linear transformations, Trans. Amer. Math. Soc. vol. 59 (1946) pp. 524-555.

2. - A structure theory for Jordan algebras, Ann. of Math. vol. 48 (1947) pp. 546-567.

3. - A theory of power-associative commutative algebras, Trans. Amer. Math. Soc. vol. 69 (1950) pp. 503-527.

4. P. M. Cohn, On homomorphic images of special Jordan algebras, Canadian Journal of Mathematics vol. 6 (1954) pp. 253-264.

5. N. Jacobson $A$ theorem on the structure of Jordan algebras, To appear in Proc. Nat. Acad. Sci. U.S.A.

\section{Ohio State University}

1 Added in proof: A Jordan ring over a field of characteristic different from two is special. See A. I. Shirshov, On special J-rings, Rec. Math. (Mat. Sbornik) N.S. vol. 38 (80) (1956) pp. 149-166. 